Dinh, Christoph; Luessi, Martin; Sun, Limin; Haueisen, Jens; Hamalainen, Matti S.:

Mne-X: MEG/EEG real-time acquisition, real-time processing, and real-time source localization framework

Zuerst erschienen in: $\quad$ Biomedical Engineering = Biomedizinische Technik. - Berlin [u.a.] : de Gruyter. - 58 (2013), Suppl. 1, Track-G, art. [4184], 2 pp.

Erstveröffentlichung: 2013-09-07

ISSN (online): $\quad$ 1862-278X

ISSN (print): $\quad$ 0013-5585

DOI: $\quad$ 10.1515/bmt-2013-4184

[Zuletzt gesehen: 2019-07-18]

„Im Rahmen der hochschulweiten Open-Access-Strategie für die Zweitveröffentlichung identifiziert durch die Universitätsbibliothek IImenau."

"Within the academic Open Access Strategy identified for deposition by IImenau University Library."

„Dieser Beitrag ist mit Zustimmung des Rechteinhabers aufgrund einer (DFGgeförderten) Allianz- bzw. Nationallizenz frei zugänglich.“

"This publication is with permission of the rights owner freely accessible due to an Alliance licence and a national licence (funded by the DFG, German

DFG

Research Foundation) respectively." 


\title{
MNE-X: MEG/EEG REAL-TIME ACQUISITION, REAL-TIME PROCESSING, AND REAL-TIME SOURCE LOCALIZATION FRAMEWORK
}

\author{
Christoph Dinh ${ }^{1,2}$, Martin Luessi ${ }^{1}$, Limin $\operatorname{Sun}^{1}{ }^{1}$ Jens Haueisen ${ }^{2,3}$ and Matti S Hamalainen ${ }^{1}$ \\ ${ }^{1}$ Athinoula A. Martinos Center for Biomedical Imaging, Massachusetts General Hospital - Massachusetts \\ Institute of Technology - Harvard Medical School, United States \\ ${ }^{2}$ Institute of Biomedical Engineering and Informatics, Ilmenau University of Technology, Germany \\ ${ }^{3}$ Biomagnetic Center, Department of Neurology, University Hospital Jena, Germany \\ chdinh@nmr.mgh.harvard.edu
}

\begin{abstract}
Providing millisecond-temporal resolution for non-invasive mapping of human brain functions, Magneto/Electroencephalography (MEG/EEG) is predestined to monitor brain activity in real-time. While data analysis to date is mostly done subsequent to the acquistion process we introduce here an acquisition and real-time analysis application. Online feedback allows the adaption of the experiment to the subject's reaction creating a whole set of new options and increasing time efficiency by shortening acquisition and offline analysis. To build a standalone application, we first designed MNE-CPP a cross-platform open source Qt5 C++ library, which implements the validated parts of our scripting toolboxes MNE-Python/MATLAB. Based on MNE-CPP we built MNE-X, which allows realtime acquisition, processing, and source localization.
\end{abstract}

Keywords: Real-Time, Magnetoencephalography, Electroencephalography, Processing, Source Localization

\section{Introduction}

Real-time processing and source localization for MEG/EEG enables possibilities for novel experimental paradigms and improves the efficacy of these methods in clinical diagnosis where fast analysis procedures are essential. Given that some information is processed during the acqusition, the subsequent off-line analysis will be facilitated and made faster. Such a real-time MEG/EEG monitor has to be able to control the MEG/EEG hardware to acquire data, as well as to be capable of processing the acquired data online. Several application frameworks $[1,2]$ to acquire as well as scripting toolboxes [2, 3, 4] to analyse MEG/EEG data already exist. For designing a standalone acquisition and processing application, such as MNE-X, it is necessary to have a highly flexible library which unites the acquisition and the analysis. Here we want to introduce MNE-CPP, our new cross-platform opensource Qt5 C++/CUDA library package. MNE-CPP is designed as a basis for standalone applications and enables the application programmer to make full use of the given hardware capabilities and develop processing pipelines with almost the ease of a scripting toolbox. MNE-CPP is part of the MNE-Suite and can be freely accessed at: https://github.com/mne-tools

\section{Methods}

MNE-CPP is a derivative of our scripting toolboxes MNE-Python/MNE-MATLAB and consists of several sublibraries (Fig. 1). Except of the inverse sub-library, MNECPP only depends on Qt5 [5] and the light-weight Eigen [6] template library for linear algebra. This enables the user to build MEG/EEG applications for almost any computational device. PCs, tablets, smartphones and even embedded solutions are supported.

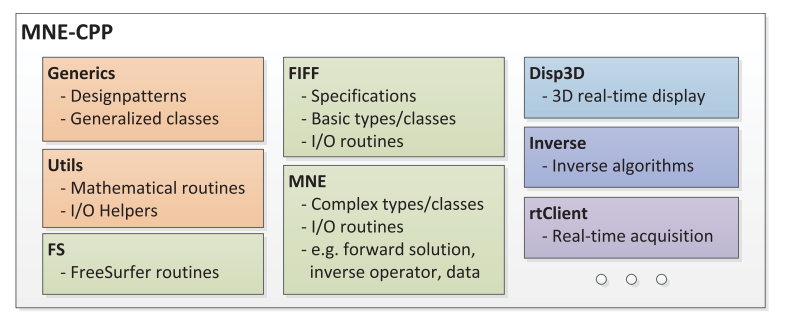

Figure 1: Sub-libraries of MNE-CPP: helpers (orange), core (green), specific tasks (blue to violet)

Besides the $\mathrm{C}++$ implementation, the inverse library provides a GPU CUDA kernel for some algorithms, and relies if GPU computing is wanted on NVDIAs nvec compiler.

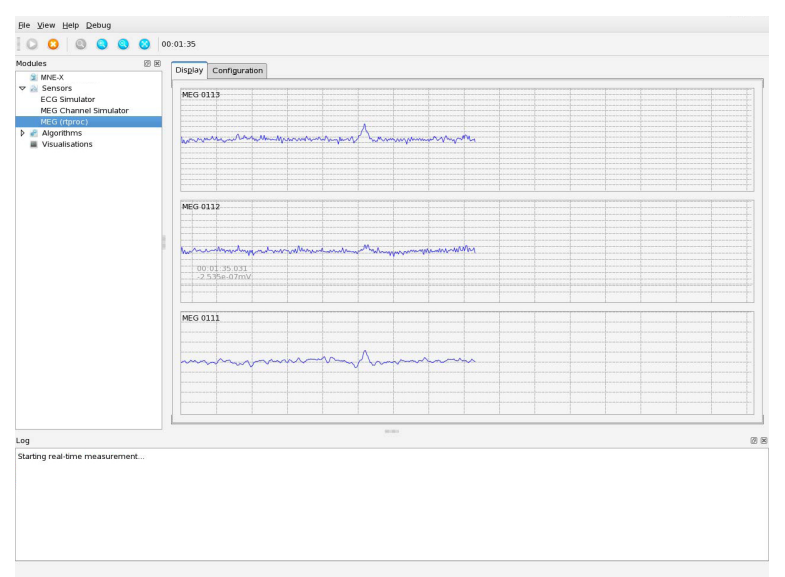

Figure 2: User interface of MNE-X 
MNE-X (Fig. 2) is built based on MNE-CPP. It is designed for the daily clinical use and comes with several real-time plug-ins, e.g. the averaging, source localization, and frequency estimation toolboxes. Also the development of further plug-ins, e.g., real-time Signal Source Separation (SSS)/ Signal Space Projection (SSP), is already in progress.

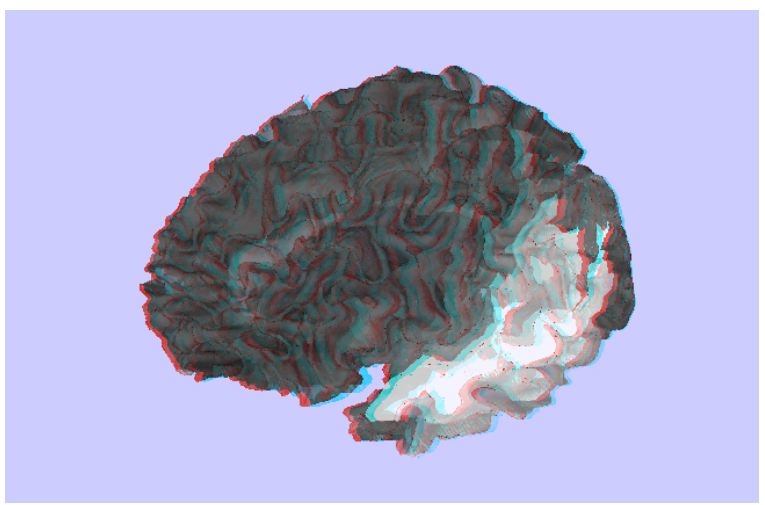

Figure 3: Screenshot of the real-time stereoscopic 3D (anaglyph red/cyan) source localization monitor. The display shows the localization of an auditory stimulus with an applied moving average.

Here we also want to introduce our online source localization toolbox, which, in addition to the localization algorithms, provides a real-time 3D stereoscopic display (fig. 3 )). The applied algorithm uses a region-of-interest forward solution clustering and an adapted MNE [7] algorithm.

\section{Results}

MNE-X is able to control the MEG/EEG hardware to acquire data. It also provides fast and effective algorithms to process the acquired data online. It is designed as a crossplatform opensource application and introduces a new plugin framework to easily adapt and extend the MEG/EEG online analyzation capabilities. To realize MNE-X we designed in a highly portable MNE-CPP library.

\section{Discussion}

By providing online feedback and increasing time efficiency, MNE-X aims to improve the clinical value of MEG/EEG by providing new experiment options and integragting them into fast diagnostic procedures. However, more work is needed to address, e.g., the accuracy of online source localization. Clinical and basic research studies are needed to assess the benefits of MNE-X and to guide its further development.

\section{Bibliography}

[1] G. Schalk, D. McFarland, T. Hinterberger, N. Birbaumer, and J. Wolpaw, "Bci2000: a general-purpose brain-computer interface (bci) system," Biomedical Engineering, IEEE Transactions on, vol. 51, no. 6, pp. 1034-1043, 2004.
[2] R. Oostenveld, P. Fries, E. Maris, and J.-M. Schoffelen, "Fieldtrip: Open source software for advanced analysis of meg, eeg, and invasive electrophysiological data," Computational Intelligence \& Neuroscience, p. 156869 (9 pp.), 20112011.

[3] A. Delorme and S. Makeig, "Eeglab: an open source toolbox for analysis of single-trial eeg dynamics including independent component analysis," Journal of Neuroscience Methods, vol. 134, no. 1, pp. 9-21, 2004.

[4] F. Tadel, S. Baillet, J. C. Mosher, D. Pantazis, and R. M. Leahy, "Brainstorm: A user-friendly application for meg/eeg analysis," Computational Intelligence \& Neuroscience, p. 879716 (13 pp.), 20112011.

[5] Qt project authors, “Qt5 cross-platform application and ui framework," 2013.

[6] Eigen authors, "Eigen 3 c++ template library," 2012.

[7] M. S. Hamalainen and R. Ilmoniemi, "Interpreting magnetic fields of the brain: minimum norm estimates," Medical \& Biological Engineering \& Computing, vol. 32, no. 1, pp. 35-42, 1994. 